\title{
Theory of the Computational Function of Microcircuit Dynamics
}

\author{
W. MAASS ${ }^{1}$ and H. MARKRAM ${ }^{2}$ \\ ${ }^{1}$ Institute for Theoretical Computer Science, Technische Universität Graz, \\ 8010 Graz, Austria \\ ${ }^{2}$ Brain and Mind Institute, Ecole Polytechnique Fédérale de Lausanne, \\ 1015 Lausanne, Switzerland
}

\begin{abstract}
This chapter discusses models for computation in cortical microcircuits in the light of general computational theories and biological data. We first review some basic concepts from computation theory. We then discuss existing models for computation in cortical microcircuits within this precise conceptual framework. We argue that models for online computing in dynamical systems require particular attention in this context, since they provide the best fit to the types of computational tasks that are solved by the brain, as well as the best fit to biological data about the anatomy and physiology of the underlying circuits.
\end{abstract}

\section{INTRODUCTION}

The neocortex has enabled a quantum leap in the ability of mammals to adapt to a rapidly changing environment by supporting the emergence of sophisticated cognitive functions. Higher cognitive function depends critically on the ability of a system to predict future events in order to generate appropriate and intelligent responses in a rapidly changing environment. The neocortex solves this computational challenge by transforming multisensory information in parallel and in real time into a multidimensional output using information that was processed and stored at almost any combination of time points in the past. Therefore, a central challenge is to understand how the neocortex is designed to solve this task and what kind of theoretical framework could explain the computational principles it uses to solve this task.

The neocortex is characterized by precise topographic maps where information from the senses is mapped onto different regions of the neocortex, and 
different regions of the neocortex are mapped back onto subcortical brain regions and onto effector organs that drive the body. Different regions of the neocortex are also intricately mapped onto each other to fuse all the modalities into a coherent perception. These topographical maps onto, between, and from the neocortex specify precisely the primary function of each cortical region; they also mean that all functions of the neocortex are interlinked. It is the manner in which these functions are interlinked that forms the cognitive architectures that allow the neocortex to construct integrated high-dimensional sensorimotor models to simulate and predict future events.

The principle of precise topographic mapping combined with massive recurrent links is applied in the neocortex not only between brain areas, but down to the most detailed level of the circuit design. The neocortex is, for example, arranged into vertical layers, each with unique afferent and efferent connectivity; this allows different brain regions to map onto different layers of the same column of neocortex. The nonspecific thalamus is mapped onto layer I, association regions onto layers II and III, specific thalamus onto layer IV, higher association areas and specific thalamus are mapped onto layer $\mathrm{V}$, and multiple brain regions as well as specific thalamic nuclei are mapped onto layer VI. In terms of output, layer II/III neurons provide the main output to association cortical regions, layer $\mathrm{V}$ provides the main output to subcortical regions and contralateral hemispheres, and layer VI provides the main output to thalamus and brain regions specializing in processing different modalities. While each layer is specialized to process primarily specific input and generate a specific output, the layers are also highly interconnected (Thomson and Morris 2002), indicating that information from multiple brain regions is interlinked in columns of neurons. It is the manner in which these layers are mapped onto each other that governs how sensory input, ongoing activity, and output are coordinated to create a coherent perception and appropriate response.

Within neocortical layers, neurons are also intricately mapped onto each other, where the anatomical and physiological properties as well as probabilities of connections between neurons are unique for each type of pre- and postsynaptic neuron combination (Gupta et al. 2000). The computational advantage of such a specific design of this recurrent microcircuit must be extremely powerful compared to a mere random recurrent network, because this intricate design is duplicated and applied throughout the neocortex in all mammalian species. Remarkable stereotypy exists in terms of morphology of cells (morphological stereotypy), electrical behavior of cells (electrophysiological stereotypy), positioning of cells (spatial stereotypy), patterning of the anatomical and physiological properties of synaptic connections between neighboring cells (local synaptic stereotypy), and in terms of long-range afferent and efferent connectivities (distal connection stereotypy) (Silberberg et al. 2002). Although there clearly exists a unique design with a considerable degree of stereotypy across different regions, ages, and species, variations are found that seem to be adaptations to the specific requirements of the brain region or species. 
The stereotypy of the neocortical microcircuit led many early anatomists to propose that neocortical neurons could be grouped anatomically into columns, roughly the diameter of the spread of the basal axonal and dendritic arbors of pyramidal neurons (about $500 \mu \mathrm{m}$ ) (see Silberberg et al. 2002). Indeed, more than $80 \%$ of the synapses of neocortical interneurons are devoted to interconnecting neurons within a diameter of $500 \mu \mathrm{m}$. Experiments in the late 1950s and early 1960s further indicated that the neocortical sheet may also be regarded functionally as being composed of small repeating columns of several thousand cells of about $500 \mu \mathrm{m}$ in diameter. Such functional modules have now been observed in many neocortical areas and in many species. More recent experiments have revealed multiple overlying functional columns, which indicate that the notion of a cortical column as a set anatomical entity is not correct. Functional modules are overlaid (Swindale et al. 2000) such that a group of neurons collaborating to perform one operation may not necessarily collaborate to perform a different operation. The dense local connectivity within a diameter of $500 \mu \mathrm{m}$, embedded in a continuous sheet of interconnected neurons, seems therefore to impart the neocortex with the remarkable ability of allowing multiple functional columns to form and overlie dynamically on the same cortical sheet. The properties of the local as well as afferent and efferent connectivity and the microcircuit continuity enable a functional neocortical column to form potentially at any point in the neocortex.

In summary, the neocortex is composed of heterogeneous microcircuits that differ with age, across brain regions, and across species. Nevertheless, many properties of the microcircuit are stereotypical, suggesting that neocortical microcircuits are merely variations of a common microcircuit template. Such a template could subserve the impressive computational capability of the neocortex, and diversification could allow microcircuits to meet the specific requirements demanded by different neocortical areas, environmental conditions, and species adaptations. The theoretical question is how such a generic template can display sufficiently powerful and versatile information-processing capabilities.

Below we introduce some basic concepts that are useful for a more rigorous discussion of this question in the context of computation theory. Thereafter we review a few existing hypotheses regarding the computational function of microcircuit dynamics in the light of various computational theories. Consequences for future research are discussed in the final section.

\section{BASIC CONCEPTS FROM THE THEORY OF COMPUTING}

We begin by defining a few basic concepts that are useful for highlighting characteristic differences among competing models for cortical computation. We refer to Savage (1998) for details regarding general computational models. Details on the computational power of artificial neural networks can be found in Sima and Orponen (2003). An elementary introduction to artificial neural networks is given in Tsodyks (2002). 


\section{Input-Output Conventions}

If all of the input for a computation is available when the computation begins, one speaks of batch input. In contrast, online computations receive a possibly "never-ending" stream of inputs and need to be able to integrate information from previously received input segments with information from the current input segment. If the output of a computation has to be delivered by a specific deadline (e.g., within $100 \mathrm{~ms}$ after a specific input segment has arrived), one calls this real-time computing. If the computational machinery can even be prompted at any time to provide its current best guess of a suitable output, without a prespecified schedule for output demands as in real-time computing, then it implements an anytime algorithm. If there exists no deadline for delivering the output of a computation, this is referred to as offline computing ${ }^{1}$.

\section{Programs, Learning, and the Difference between Computing and Learning}

The program of a computational model specifies which algorithm is applied to what type of input, and where and when the output of a computation is provided. One usually contrasts fully programmed computational models (such as Turing machines or cellular automata) with models that are able to learn (such as multilayer perceptrons with backprop). One usually says that a machine (or organism) learns when information contained in current inputs affects the way in which it processes future inputs. Of course, the way in which current inputs can change future processing has to be prespecified by a learning algorithm. Thus a computational device that learns also requires a program - one on a higher level that specifies the organization of learning for the system (i.e., how the handling of future inputs is affected by current or preceding inputs). Even "self-organizing" computational models require such higher-level programs (which might, of course, be encoded through distributed local operating rules).

1 Offline computing in combination with batch input is the most common mode considered in computational complexity theory, where upper bounds are provided for the number of computation steps that a specific algorithm needs on a specific computational model for completing its computation for any input of length $n$. Such upper bounds are usually expressed by terms that may, for example, have the form $c_{1} \cdot n^{d}+c_{2}$ with arbitrary constants $c_{1}, c_{2}$ (abbreviated $O\left(n^{d}\right)$ ) that absorb all finite size effects into the constants $c_{1}$ and $c_{2}$. In the more general parts of computational complexity theory, one focuses on the exponent $d$. For example, the best-known complexity class consists of all families of computational problems for which there exists a (deterministic) algorithm and some finite exponent $d$ so that any instance of this computational problem of any length $n$ can be solved on a Turing machine within time $O\left(n^{d}\right)$ by this algorithm. Although this complexity class $P$ is frequently viewed as a characterization of the universe of computationally solvable problems, one has to be careful in applications of these asymptotic concepts to scenarios such as computations in the brain, where it is clear that only inputs of length $n \leq n_{0}$ (for some specific finite bound $n_{0}$ ) can occur, and finite size effects that determine, for example, the size of the previously mentioned constants $c_{1}$ and $c_{2}$ may become quite relevant. It should also be mentioned that the class $P$, as well as most other concepts from classical computational complexity theory, are only meaningful for offline computations on batch input. 
The preceding definitions are only meaningful for computations on batch inputs. The analysis of learning machines (or organisms that learn) becomes conceptually more difficult when one looks at online computing, since there one cannot talk about "current" and "future" inputs, one just has a (virtually) endless input stream. One could, of course, talk about current and future segments of this input stream, but even very simple devices for online computing, such as linear filters or finite automata, have the property that current input segments will influence the way in which future input segments will be processed, without any "learning" or "adaptation" being involved. Phrased differently, for any model of online computing that integrates information from several preceding input segments for its current output (i.e., for any model that is capable of temporal integration of information), it becomes difficult or even impossible to distinguish computing conceptually from learning. The "difference" becomes usually a matter of perspective, where, for example, online computations that take place on larger timescales, or which involve higher levels of computational organization, might be referred to as learning.

\section{Internal States and Transitions between States}

The internal state of a computational model (or of an autonomously learning system) at time $t$ should contain all information that would have to be stored if one interrupts its processing at time $t$ for a while and then wants to restart it and continue its operation at a later time $t+\Delta$ based on the information contained in this stored information, as if there had been no interruption. Such internal states can be digital or analog and can form finite or infinitely large sets of (possible) internal states. A finite automaton (or finite state machine) is characterized by the fact that it only has a finite state set, whereas a Turing machine (which is basically just a finite automaton together with a read/write tape) has an infinite set of digital states, since its current tape inscription (whose length is finite at any given time $t$, but may grow with $t$ ) is also part of its current internal state.

Transitions between internal states can be deterministic or stochastic. In the deterministic case, a program determines transitions between internal states (dependent on the current state and current input). In the case of stochastic computations, a program specifies the probability that a particular state $s$ is assumed at time $t+1$, given the state assumed at time $t$ and the current input.

For computational models that work in continuous time, one commonly writes the program in the form of a differential equation. The resulting computational models are referred to as dynamical systems.

\section{Computational Goals}

Computational goals may be very specific, for example, to multiply two numbers or, more general, such as predicting future inputs or surviving for a long 
time in a complex real environment with limited resources. Very specific computational goals, as in the first example, are characteristic of our current use of digital computers, whereas more general computational goals are potentially more characteristic of the brain.

\section{Examples of Computational Models}

Turing machines are computational models that are particularly suited for studying deterministic offline computations on digital batch input in discrete time with a number of internal states, which is finite at any time $t$, but may grow unboundedly with $t$. Turing machines are universal in the sense that one has not been able to come up with any digital computation that cannot be carried out by a Turing machine.

Digital or analog feedforward circuits (e.g., feedforward Boolean circuits or feedforward neural nets) constitute another class of standard models for offline computing. They can also be used for real-time computing (in the form of pipelining) since their computation time on any (batch) input is limited by the depth of the circuit. However, they can only be used for those computations on sequences of inputs where no temporal integration of information from several successively presented batch inputs is required.

Finite automata are computational models with a fixed finite set of internal states that are suitable for online computations in discrete time; in fact, they are perfectly suited for real-time computing. Their current state can hold information about current and past inputs, and their state transitions can be deterministic or stochastic. Cellular automata are ensembles of infinitely many identical copies of some finite automaton located on the nodes of some infinite graph, where every node has the same finite number of neighbors (e.g., a two-dimensional grid). At every discrete time step, each automaton changes its state and determines the output to its direct neighbors dependent on the inputs that it has received from its direct neighbors at the end of the preceding time step. ${ }^{2}$ The input to a cellular automaton is commonly encoded in the initial states of the individual finite automata. It is well known that every Turing machine (hence any currently existing digital computer) can be simulated by some cellular automaton. ${ }^{3}$

Artificial neural networks are also usually considered only with discrete time, but with analog internal states (so that even a single internal state may contain infinitely many bits of information). Both deterministic and stochastic state transitions are considered. Feedforward neural nets are primarily used for

2 The well-known "Game of Life" is an example of such a cellular automaton.

3 To be precise, this holds only for a cellular automaton consisting of infinitely many cells. A cellular automaton consisting of just finitely many cells can only simulate those Turing machine computations that can be carried out within a corresponding space bound. Basic results from computational complexity theory imply that the computational power of Turing machines (and hence also of cellular automata) does not saturate at any finite space bound. 
offline computing since they cannot integrate information from successive inputs; however, recurrent neural nets are suitable for offline and online computations. Although the learning capability of artificial neural networks is viewed as one of their essential properties, the organization of learning for neural networks is usually not controlled by the network itself, but by an external supervisor. ${ }^{4}$ From that perspective neural network models are far away from being autonomously learning systems.

Genetic (or evolutionary) algorithms are programs for computational models with stochastic state transitions in discrete time whose computational goal is the generation of formal objects ("agents") that have high "fitness" according to a fitness function that is part of the program.

\section{OPTIONS FOR UNDERSTANDING THE COMPUTATIONAL FUNCTION OF MICROCIRCUIT DYNAMICS}

Obviously the computational function of the brain is to enable an autonomous system to survive in the real world. Often the computational function of the brain is seen more narrowly, conceptually separating computing from learning. However, as discussed in the preceding section, such distinction is not very meaningful for analyzing online computations on input streams (only for isolated computations on batch inputs). ${ }^{5}$ Hence one needs to understand learning as an integral part of the computational function of neural microcircuits.

In the subsequent subsections, we will review three categories of computational models for neural microcircuits that differ with regard to the type of computation that is supported by these models (offline vs. online computing) and with regard to the circuit structures on which they focus (feedforward vs. recurrent circuits). In addition, the models also differ with regard to their underlying assumption about (a) the computational specialization of cortical microcircuits (Do cortical microcircuits exist that carry out just one particular computation?), and (b) how the computational function is "programmed" into cortical microcircuits (Is their computational function directly genetically programmed or acquired by learning in the course of genetically programmed developmental procedures and fine-tuned by synaptic plasticity throughout adulthood?).

4 For example, the selection of training examples of a learning rate or of the time points when learning is turned on and off are typically taken over by a human supervisor, and are not carried out by the "learning device" (e.g., a multilayer perceptron) itself. In that sense, a multilayer perceptron with backprop cannot be viewed as an autonomously learning machine.

5 Separate trials in neurophysiological experiments often aim at testing an organism on artificially created batch inputs and proceed on the assumption that one can ignore the fact that from the perspective of the organism they still constitute segments of one continuous input stream. 


\section{Microcircuits as Modules That Compute Stereotypical Basis Functions for Computations on Batch Input}

One can compute any Boolean function (i.e., any function $f:\{0,1\}^{m} \rightarrow\{0,1\}^{n}$ for arbitrary $m, n \in \mathbf{N})^{6}$ on a feedforward circuit consisting of units or subcircuits that compute certain stereotypical basis functions. For example, it suffices to have subcircuits that compute an OR of two input bits in conjunction with subcircuits that compute a negation. It even suffices to iterate stereotypical copies of a single subcircuit, for example, of a subcircuit that computes ( $x_{i}$ AND NOT $x_{2}$ ). From a mathematical point of view there is nothing special about such basis functions, and many different sets of basis functions exist that are complete in the sense that all Boolean functions can be generated by them.

For analog computations it is more meaningful to look at ways of approximating (rather than computing) arbitrary given functions $f:[-B, B]^{m} \rightarrow[-B, B]^{n}$ from real numbers into real numbers by circuits that are composed of stereotypical subcircuits that compute suitable basis functions. Again there exist many different sets of basis functions that are complete in the sense that any continuous function $f:[-B, B]^{m} \rightarrow[-B, B]^{n}$ can be approximated arbitrarily closely ${ }^{7}$ through suitable combinations of such basis functions. Since continuous functions can be approximated arbitrarily closely by polynomials (on any bounded domain $\left.[-B, B]^{m}\right)$, it suffices, for example, to choose addition and multiplication of real numbers (in combination with real or rational constants) as basis functions. The universal approximation theorem from artificial neural networks states that one can also choose as basis functions sigmoidal gates applied to weighted sums of the inputs, that is, functions of the form:

$$
\sigma\left(\sum_{i=1}^{k} w_{i} x_{i}\right) \text { with } \sigma(x)=\frac{1}{1+e^{-x}} .
$$

In fact, one can use here instead of the sigmoidal function $\sigma$ almost any nonlinear function $h=\mathbf{R} \rightarrow \mathbf{R}$. As an alternative it suffices to use a single application of a winner-take-all-like nonlinearity in combination with subcircuits that compute just linear weighted sums (Maass 2000). Thus we see that also for the computation of analog functions there exist many different sets of basis functions that are complete.

A tempting hypothesis regarding the computational role of cortical microcircuits is that there exist genetically programmed stereotypical microcircuits that compute certain basis functions. Numerous ways in which circuits of

$6\{0,1\}^{m}$ is the set of all bit strings of length $m$. $[-B, B]$ is the set of all real numbers with absolute value bounded by $B$.

7 This means that for any given $\varepsilon$ there exist such approximating function $C$ so that $\|f(\underline{x})-C(\underline{x})\| \leq \varepsilon$ for any $\underline{x} \in[-B, B]^{m}$, where $\|f(\underline{x})-C(\underline{x})\|$ denotes the Euclidean distance between the points $f(\underline{x})$ and $C(\underline{x})$ in the $n$-dimensional space $\mathbf{R}^{n}$. 
neurons can potentially compute a complete set of Boolean basis functions have been proposed (see, e.g., Shepherd and Koch 1998). For example, a single shunting synapse can in principle compute the Boolean function $\left(x_{i}\right.$ AND NOT $x_{2}$ ), which forms a complete basis. Also many possible ways in which single neurons or circuits of neurons can potentially compute basis functions for analog computing (e.g., addition and multiplication) have been collected in Table 1.2 of Shepherd and Koch (1998), which is reproduced in this chapter (see Table 18.1), and in Chapter 21 of Koch (1999).

A possible way in which circuits of neurons could implement a sigmoidal gate has been proposed in Maass (1997). In Pouget and Sejnowski (1997) products of a Gaussian function (e.g., of the retinal location of a cue) and a sigmoidal function (e.g., of eye position) are proposed as basis functions for sensorimotor transformations. They prove that these basis functions are complete in the sense

Table 18.1 Potential computational functions of various biophysical mechanisms in neural circuits (from Shepherd 1990; reprinted with permission of Oxford University Press).

\begin{tabular}{|c|c|c|c|}
\hline $\begin{array}{l}\text { Biophysical } \\
\text { Mechanism }\end{array}$ & $\begin{array}{l}\text { Neuronal } \\
\text { Operation }\end{array}$ & $\begin{array}{l}\text { Example of } \\
\text { Computation }\end{array}$ & Timescale \\
\hline $\begin{array}{l}\text { Action potential initia- } \\
\text { tion }\end{array}$ & $\begin{array}{l}\text { Threshold, one-bit ana- } \\
\text { log-to-digital converter }\end{array}$ & & $0.5-5 \mathrm{~ms}$ \\
\hline $\begin{array}{l}\text { Action potentials in } \\
\text { dendritic spines }\end{array}$ & $\begin{array}{l}\text { Binary OR, AND, } \\
\text { AND-NOT gate }\end{array}$ & & $0.1-5 \mathrm{~ms}$ \\
\hline $\begin{array}{l}\text { Nonlinear interaction } \\
\text { between excitatory and } \\
\text { inhibitory synapses }\end{array}$ & $\begin{array}{l}\text { Analog AND-NOT } \\
\text { veto operation }\end{array}$ & $\begin{array}{l}\text { Retinal directional selec- } \\
\text { tivity }\end{array}$ & $2-20 \mathrm{~ms}$ \\
\hline $\begin{array}{l}\text { Spine-triadic synaptic } \\
\text { circuit }\end{array}$ & $\begin{array}{l}\text { Temporal differentia- } \\
\text { tion high-pass filter }\end{array}$ & $\begin{array}{l}\text { Contrast gain control in } \\
\text { the LGN }\end{array}$ & $1-5 \mathrm{~ms}$ \\
\hline Reciprocal synapses & Negative feedback & $\begin{array}{l}\text { Lateral inhibition in olfac- } \\
\text { tory bulb }\end{array}$ & $1-5 \mathrm{~ms}$ \\
\hline $\begin{array}{l}\text { Low, threshold calcium } \\
\text { current }\left(I_{\mathrm{T}}\right)\end{array}$ & Triggers oscillations & $\begin{array}{l}\text { Gating of sensory informa- } \\
\text { tion in thalamic cells }\end{array}$ & $5-15 \mathrm{~Hz}$ \\
\hline NMDA receptor & AND-NOT gate & Associative LTP & $0.1-0.5 \mathrm{~s}$ \\
\hline $\begin{array}{l}\text { Transient potassium } \\
\text { current }\left(I_{\mathrm{A}}\right)\end{array}$ & Temporal delay & $\begin{array}{l}\text { Escape reflex circuit in } \\
\text { Tritonia }\end{array}$ & $10-400 \mathrm{~ms}$ \\
\hline $\begin{array}{l}\text { Regulation of potassium } \\
\text { currents }\left(I_{\mathrm{M}}, I_{\mathrm{AHP}}\right) \text { via } \\
\text { neurotransmitter }\end{array}$ & Gain control & $\begin{array}{l}\text { Spike frequency accom- } \\
\text { modation in sympathetic } \\
\text { ganglion and hippocampal } \\
\text { pyramidal cells }\end{array}$ & $0.1-2 \mathrm{~s}$ \\
\hline $\begin{array}{l}\text { Long-distance action of } \\
\text { neurotransmitters }\end{array}$ & $\begin{array}{l}\text { Routing and addressing } \\
\text { of information }\end{array}$ & & $1-100 \mathrm{~s}$ \\
\hline Dendritic spines & $\begin{array}{l}\text { Postsynaptic modifica- } \\
\text { tion of functional con- } \\
\text { nectivity }\end{array}$ & Memory storage & $\infty$ \\
\hline
\end{tabular}


that weighted sums of such functions can approximate any desired continuous sensorimotor transformation. It is argued that the outputs of some parietal neurons can be approximated quite well by such basis functions. However, it is not known exactly how the computation of these basis functions is neurally implemented. A more general perspective of this basis function approach is given in Salinas and Sejnowski (2001).

Most approaches based on static basis functions do not provide good models for the biologically more realistic case of online computing on time-varying input streams. Furthermore, there are no good models for explaining how the composition of basis functions to larger computational modules is organized or learned by neural systems.

\section{Microcircuits as Dynamical Systems Whose Input Is Encoded in the Initial State}

Complementary approaches towards understanding the role of stereotypical cortical microcircuits for cortical computation emphasize that these microcircuits are synaptically connected in a highly recurrent manner, not in the way of a feedforward circuit. Thus, we will now focus on recurrent circuits. In this subsection, however, we discuss only approaches that focus on offline computations, that is, computations on batch input that are encoded in the initial state of the system. Turing machines fall into this category, but also cellular automata, recurrent neural networks (e.g., Hopfield nets, attractor neural networks), and other more general dynamical systems are traditionally considered in this offline computational mode. ${ }^{8}$ In fact it appears that the majority of computational models currently considered in computational neuroscience fall into this category.

Recurrent circuits can be composed from the same basis functions as feedforward circuits (see previous section). However, a new problem arises. In a feedforward circuit, the computational control that determines which subcircuit is activated at any given moment is implicitly encoded by the underlying wiring diagram, which is in that case a directed acyclic graph. Computations in recurrent circuits that implement Turing machines, cellular automata, or recurrent neural nets in discrete time require a central clock in conjunction with a protocol that determines which subcircuit is active at which clock tick. Although such computational organization is theoretically also possible for a circuit consisting of integrate-and-fire neurons (Maass 1996), the biologically more realistic case is obviously that of computing in continuous time with the interaction between computing elements described by differential equations. In this way one arrives at special cases of dynamical systems. The (batch) input is traditionally encoded in the initial state of the dynamical system, and one usually waits until it has converged to an attractor, which could be a fixed point, a limit cycle, or a "strange

8 The latter models can also be used for ting; such less traditional uses will be discussed in the next subsection. 
attractor" as considered in chaos theory. Even dynamical systems that are composed of very simple dynamic units (e.g., sigmoidal gates) can have very complicated dynamics, and it is not clear what particular contribution should be expected from cortical microcircuits if they are viewed as implementations of such dynamic units. Freeman (1975) proposed using a conceptual framework by Ketchalsky for classifying components of recurrent circuits (K0, KI, KII sets, etc.), but the nature of nonlinear recurrent circuits tends to be in the way of any meaningful decomposition into simpler components.

Synfire chains (Abeles 1991) have been proposed as potential dynamic modules of complex recurrent circuits that inject a particular timing structure into the recurrent circuit. This is theoretically possible since a synfire chain (in its most basic form) is a feedforward circuit whose subsets of neurons are activated in a sequential manner. However, no significant computations can be carried out by a single synfire chain. It has been conjectured that interactions between several overlapping synfire chains may attain significant computational power, but this has not yet been demonstrated (without postulating an "intelligent" higher-order structure). Obviously, one needs to find principles by which a network of synfire chains could be autonomously created, structured, and updated. Another interesting open problem is how such a network could learn to process time-varying input streams.

\section{Microcircuits as Generic Modules for Online Computing in Dynamical Systems}

We now consider models for the computational function of cortical microcircuits that allow them to carry out online computations on complex input streams (which requires temporal integration of information). Finite automata are capable of carrying out such computations, although only on digital inputs in discrete time. To implement an arbitrary finite automaton, it suffices to combine a feedforward Boolean circuit that computes the transition function between states with computational units that act as registers for storing and retrieving information. One possible neural implementation of such registers was proposed in Douglas et al. (1995), using hysteretic effects in recurrent circuits. Altogether the main weakness of finite automata as a conceptual framework for neural computation is their strongly digital flavor (discrete time and discrete states), which makes learning or adaptation (that in neural systems usually involves gradient descent in one form or another, except for genetic algorithms) less powerful in this context.

Another attractive conceptual framework for the analysis of neural computation on online input streams is provided by linear and nonlinear filters. Numerous biophysical mechanisms that implement specific linear and nonlinear filters have been identified (see Table 18.1; Shepherd and Koch 1998; Koch 1999). Marmarelis and Marmarelis (1978) introduced into neurophysiology a number 
of useful techniques for modeling the input-output behavior of black-box neural circuits by linear and nonlinear filters. A more recent account is given in Rieke et al. (1997). These techniques rely on Volterra series (or equivalently Wiener series) as mathematical frameworks for modeling online computations of neural systems on continuous input streams or spike trains as inputs. Volterra series model the output of a system at time $t$ by a finite or infinite sum of terms of the form:

$$
\int_{0}^{\infty} \ldots \int_{0}^{\infty} h_{d}\left(\tau_{1}, \ldots, \tau_{2}\right) \cdot u\left(t-\tau_{1}\right) \cdot \ldots \cdot u\left(t-\tau_{d}\right) d \tau_{1} \ldots d \tau_{d},
$$

where some integral kernel $h_{d}$ is applied to products of degree $d$ of the input stream $u(\cdot)$ at various time points $t-\tau_{i}$ back in the past. Usually only linear $(d=$ $1)$ or quadratic $(d=2)$ filters are used for modeling specific neural systems, since too many data would be needed to fit higher-order kernel functions $h_{d}$. Not all possible computations on input streams $u(\cdot)$ can be modeled by Volterra series (of any degree), since any Volterra series (with convergent integral terms) has automatically a fading memory, where features of the input streams $u(\cdot)$ at any specific time point in the past have decreasing influence on the current output at time $t$ when $t$ grows. In addition, a Volterra series can only model outputs that depend in a smooth manner on the input stream $u(\cdot)$. Thus they can, for example, model spike output only in the form of smoothly varying firing rates or firing probabilities. On the other hand this mathematical framework imposes no constraint on how slowly the memory fades and how fast the smoothly varying output changes its value.

This conceptual framework of filters, which has traditionally been used primarily for analyzing signal processing rather than computing, was recently used by Maass and Sontag (2000) and Maass et al. $(2002,2004)$ as the basis for a new approach towards understanding the computational function of microcircuit dynamics. The liquid state machine was introduced as a generalization of the model of a finite automaton to continuous time and continuous ("liquid") states (see Figure 18.1).

To make this model better accessible to learning than the finite automaton, it was postulated that the liquid states (i.e., that part of the current state of the circuit that is expressed in its spiking activity and, therefore, "visible" for readout neurons) generated by a neural microcircuit should contain sufficient information about the recent input stream $u(\cdot)$ that other neurons ("readout neurons") have to learn in order to select and recombine those parts of the information stream contained in the time-varying liquid state useful for their specific computational task. Here, it is natural to apply a variant of the basis function idea discussed earlier and look for possible characterizations of sets of basis filters (that could be implemented, for example, by specific components of cortical microcircuits), which endow the resulting liquid states with the capacity to absorb 


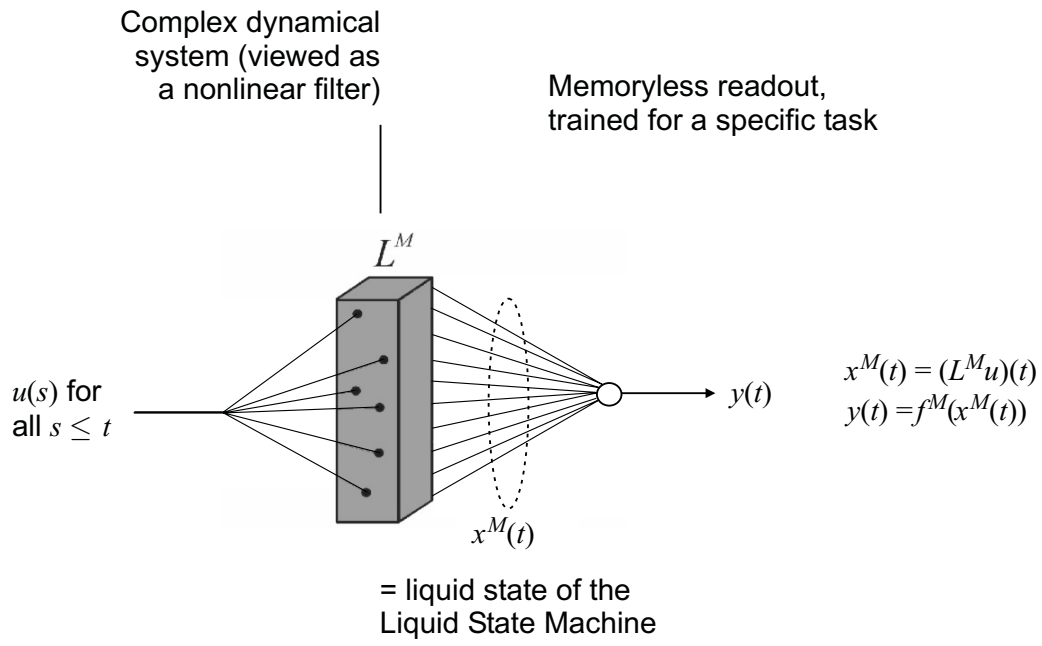

Figure 18.1 Structure of a liquid state machine, which transforms input streams $u(\cdot)$ into output streams $y(\cdot)$.

enough information about the input stream $u(\cdot)$. A mathematical theorem (Maass et al. 2002) guarantees that sufficient diversity of the basis filters implemented by the components of a neural microcircuit, for example, neurons or dynamic synapses with sufficiently diverse time constants (Maass and Sontag 2000), suffices to endow the resulting liquid state machine with the capability to approximate in principle any input-output behavior that could potentially be approximated by a (finite or infinite) Volterra series. The available amount of diversity in a microcircuit can be measured indirectly via its separation property (Maass et al. 2002).

Another potential computational function of microcircuit dynamics arises if one considers such a liquid state machine from the perspective of an (approximately) linear readout neuron, for which it should become feasible to learn to select and recombine those aspects of liquid states that may be needed for specific tasks (e.g., smooth eye pursuit, or classification and prediction of dynamic visual scenes). A microcircuit can boost the capability of any linear readout by adding a certain redundancy to the information contained in its stream of liquid states, for example, also precomputing nonlinear combinations of salient timevarying variables (analogously as in the special case of gain fields), see Figure 18.2. Very recently, in Maass, Legenstein et al. (2005), a general quantitative method was developed to evaluate such kernel capability of neural microcircuits (where the notion of a kernel is used here in the sense of support vector machines in machine learning - it goes back to the idea of a fixed nonlinear preprocessing proposed already in the 1950s by Rosenblatt's work on perceptrons, but applied here to a time-varying context). Forthcoming new results show that the computational power of the resulting computational model becomes 


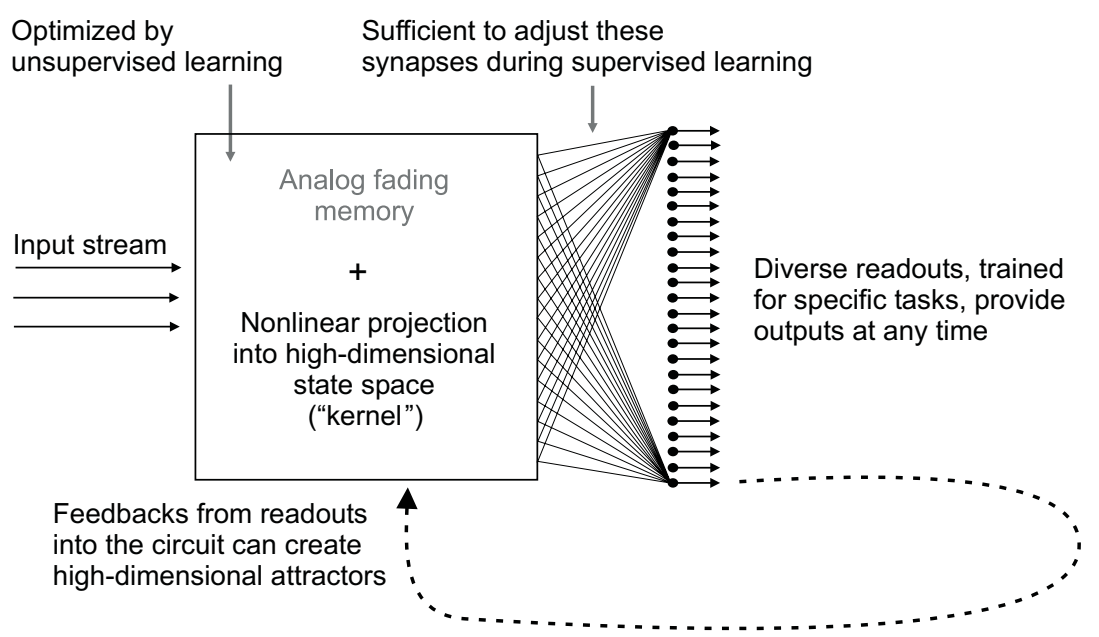

Figure 18.2 A system-oriented interpretation of the computational function of microcircuit dynamics.

significantly larger if feedback from trained readouts back into the circuit is also taken into account, as indicated by the dashed line in Figure 18.2 (Maass, Joshi et al. 2005).

From this perspective a cortical microcircuit is not viewed as an implementation of a single computation, but as a more universal computational device that can support simultaneously a large number of different computations. An example is given in Figure 18.3, where 7 different linear readouts from a generic neural microcircuit model consisting of 270 neurons had been trained to output at any time the result of 7 different computational operations on information provided to the circuit in the form of 4 spike trains (a sample is shown at the top of Figure 18.3). After training the weights of these linear readouts had been fixed. The results shown in Figure 18.3 are for new input spike trains that had never before been injected into the circuit, thereby demonstrating good generalization capability of this simple learning scheme (see Maass et al. 2002 for details).

Several experimental studies in the group of Yves Frégnac have shown that neurons can in fact be trained via current injections (even in adult animals in vivo) to read out particular aspects of the "liquid state" represented by the current firing activity of presynaptic neurons (see, e.g., Debanne et al. 1998). However, it has remained open by which principles readout neurons can be trained autonomously within a neural system to perform such task. This is least dubious in the case of prediction learning, where the arrival of the next input could provide such current injection into a readout neuron that learns to perform such prediction task as in the experiments of Debanne et al. (1998). These experimental data can be explained on the basis of standard rules for spike-timing-dependent plasticity (STDP) (see Legenstein et al. 2005). Another quite realistic scenario is 


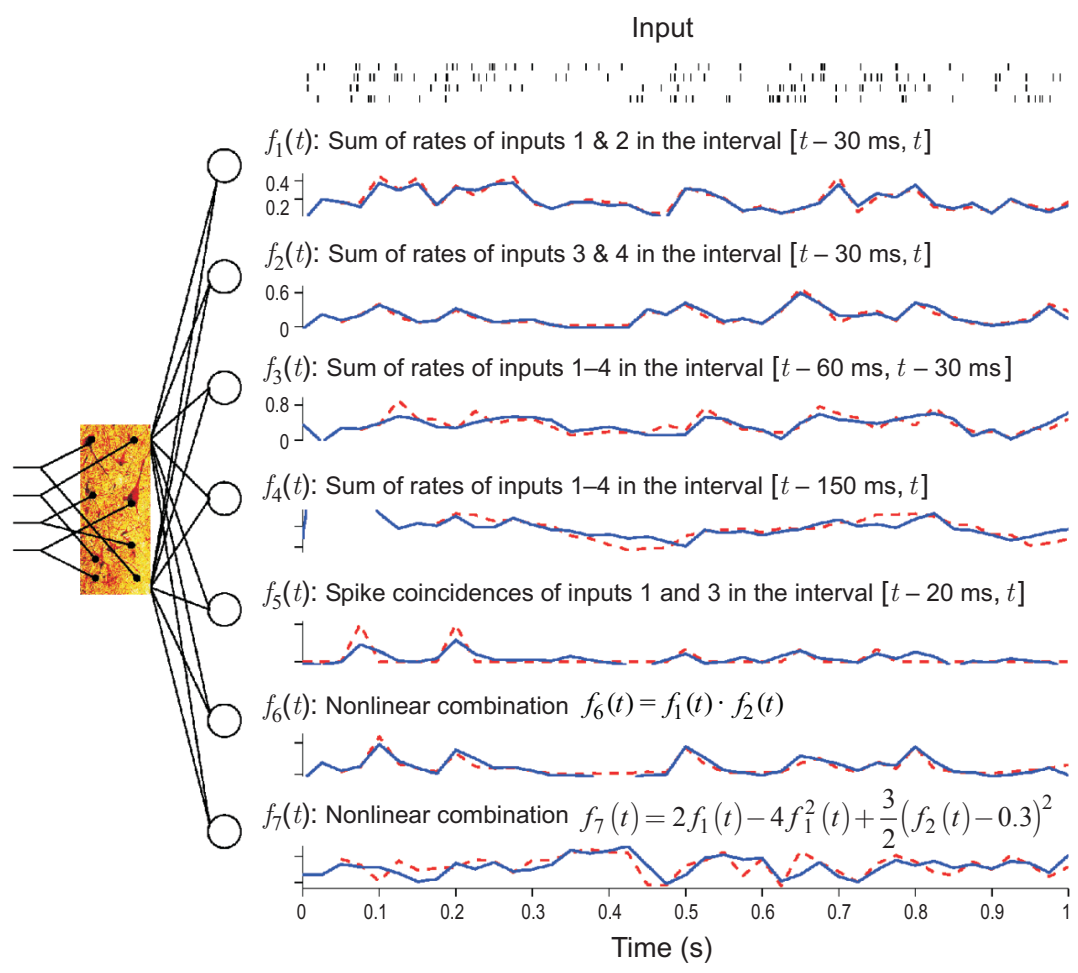

Figure 18.3 Multi-tasking in real time. Input spike trains were randomly generated in such a way that at any time $t$ the input contained no information about input rates that were used more than $30 \mathrm{~ms}$ ago. Firing rates $r(t)$ were randomly drawn from the uniform distribution over $(0 \mathrm{~Hz}, 80 \mathrm{~Hz})$ every $30 \mathrm{~ms}$, and input spike trains 1 and 2 were generated for the present $30 \mathrm{~ms}$ time segment as independent Poisson spike trains with this firing rate $r(t)$. This process was repeated (with independent drawings of $r(t)$ and Poisson spike trains) for each $30 \mathrm{~ms}$ time segment. Spike trains 3 and 4 were generated in the same way, but with independent drawings of another firing rate $\widetilde{r}(t)$ every $30 \mathrm{~ms}$. The results shown in this figure are for test data that were never before shown to the circuit. Below the 4 input spike trains the target (dashed curves) and actual outputs (solid curves) of 7 linear readout neurons are shown in real-time (on the same time axis). Targets were to output every $30 \mathrm{~ms}$ the actual firing rate (rates are normalized to a maximum rate of $80 \mathrm{~Hz}$ ) of spike trains 1 and 2 during the preceding $30 \mathrm{~ms}\left(f_{1}\right)$, the firing rate of spike trains 3 and 4 $\left(f_{2}\right)$, the sum of $f_{1}$ and $f_{2}$ in an earlier time interval $(t-60 \mathrm{~ms}, t-30 \mathrm{~ms})\left(f_{3}\right)$ and during the interval $(t-150 \mathrm{~ms}, t)\left(f_{4}\right)$, spike coincidences between inputs 1 and $3\left(f_{5}(t)\right.$ is defined as the number of spikes that are accompanied by a spike in the other spike train within $5 \mathrm{~ms}$ during the interval $(t-20 \mathrm{~ms}, t))$, a simple nonlinear combination $f_{6}$, and a randomly chosen complex nonlinear combination $f_{7}$ of earlier described values. Since all readouts were linear units, these nonlinear combinations are computed implicitly within the generic microcircuit model. Average correlation coefficients between targets and outputs for 200 test inputs of length $1 \mathrm{~s}$ for $f_{1}$ to $f_{7}$ were $0.91,0.92,0.79,0.75,0.68,0.87$, and 0.65 . 
the case of association learning, where a "teaching current" could be injected into a readout neuron by projection neurons from other microcircuits in cortical or subcortical structure that are involved in processing inputs from other sensory modalities, or which signal external or internal rewards.

An attractive feature of this computational framework is that it produces a possible explanation for the anytime computing capabilities of neural systems, since readouts can learn to transform, at any moment in time, the currently provided liquid state into the best guess for a decision or a parameter value that is needed for their specific task. Another interesting aspect is that this approach is compatible with biological data regarding oscillations that are superimposed on sensory inputs (Kaske and Maass 2005). Häusler and Maass (2005) have also shown that this approach can be applied to more detailed cortical microcircuit models with data-based connectivity between cortical layers.

This approach also provides a computational explanation for the large-scale architecture of the brain, where sensory inputs and internal outputs are not spread out uniformly all over the brain (see INTRODUCTION). Rather, each brain area is characterized by the specific set of information streams that it receives. This feature is a prerequisite for online computing with dynamical systems, since different input streams that converge onto a single microcircuit all have an influence on its internal dynamics, thereby facilitating computations that depend on segments of all these information streams. Some other input stream that is not relevant for these computations would influence the microcircuit dynamics as well, but would represent a huge source of noise from the perspective of these computations, in particular blowing up and possibly interleaving the classes of equivalent circuit states (see Maass et al. 2004, Section 5) that a readout neuron has to learn to distinguish.

An essentially equivalent computational framework to liquid computing, echo state networks, has been developed independently in an engineering context (Jäger 2002), and currently surpasses all other known methods for various time series predition and adaptive nonlinear filtering tasks (Jäger and Haas 2004). Other recent work relates these approaches to earlier work by Chris Langton et al. on computation on the edge of chaos in dynamical systems (for a review, see Legenstein and Maass 2005a, b), but applied now to anytime computing on continuous input streams rather than to offline computations on static batch input (Bertschinger and Natschläger 2004). It is argued there that neither the "ordered" nor the "chaotic" regime of recurrent circuits (where recurrent circuits are viewed here as special cases of dynamical systems) are well suited for computing, but rather the regime in between (the "edge of chaos"). This has been demonstrated by Bertschinger and Natschläger (2004) for synchronized recurrent circuits consisting of threshold gates. Results of Maass, Legenstein et al. (2005) suggest that for more realistic models of neural microcircuits the "edge of chaos" becomes harder to conceptualize, and they propose measuring the computational power and generalization capability of neural microcircuits 
instead by a quantitative measure of its kernel quality and a direct estimate of its generalization capability (VC-dimension).

\section{Other Approaches}

Various other approaches exist for explaining or modeling biological neural computation, which have not yet given rise to specific hypotheses regarding the computational role of cortical microcircuits. A number of interesting brain theories are based on information theoretic concepts, such as redundancy reduction, the information bottleneck method, and theories of optimal neural coding. Another family of models for biological neural computation uses generative models, which are based on the hypothesis that the large-scale computational goal of cortical computation is to reconstruct sensory inputs, and to "explain" them as being generated by independent components or factors that can be learnt in an unsupervised manner from the statistics of the inputs. An attractive feature of this approach is that it gives rise to autonomously learning systems. However, in a concrete biological context it is hard to demonstrate or even make precise the theoretically very attractive goal of reconstructing the input in terms of internally represented "hidden" sources. For example, top-down connections to primary sensory cortices appear to contribute to purpose-related interpretations of raw sensory input, rather than to the reconstruction of a (usually dynamically varying) sensory input; see, for example, Chapter 45 of Chalupa and Werner (2004) for the case of visual input. Unfortunately, the biologically more realistic goal of predicting future inputs (rather than reconstructing preceding inputs) has not yet given rise to an equally attractive theoretical approach.

\section{DISCUSSION}

This short survey shows that there exist drastic differences regarding theories on the computational function of cortical microcircuits. To find the most appropriate computational theory for a neural microcircuit one first needs to decide whether this circuit is designed to carry out offline computations on batch inputs or online computations on input streams. One also needs to decide to what extent this circuit is genetically programmed to carry out one specific computational operation, or whether it is designed to provide numerous and diverse "neural users" with a suitably processed amalgamation of the input streams that enter this microcircuit, where the specific output delivered to any particular "neural user" is to some degree shaped by learning.

The computational function of various salient aspects of cortical microcircuits is presently still not known, especially the computational role of specific cortical layers with particular types of neurons that are connected by particular types of synapses with particular probabilities with other specific types of 
neurons on specific layers. Another big open problem is the organization of learning in cortical microcircuits; for example, we need to know how the plasticity of its synapses is gated, and what other processes regulate the computational function of its neurons in dependence of their individual history and the statistics of their inputs.

\section{ACKNOWLEDGMENTS}

Written under partial support by the Austrian Science Fund FWF, project P15386 and S9102-N04, PASCAL, project IST2002-506778, and FACETS project FP6-015879 of the European Union.

\section{REFERENCES}

Abeles, M. 1991. Corticonics: Neural Circuits of the Cerebral Cortex. Cambridge: Cambridge Univ. Press.

Bertschinger, N., and T. Natschläger. 2004. Real-time computation at the edge of chaos in recurrent neural networks. Neural Comput. 16:1413-1436.

Chalupa, L.M., and J.S. Werner. 2004. The Visual Neurosciences. Cambridge, MA: MIT Press.

Debanne, D., D.E. Shulz, and Y. Fregnac. 1998. Activity dependent regulation of on- and off-responses in cat visual cortical receptive fields. J. Physiol. 508:523-548.

Douglas, R.J., C. Koch, M. Mahowald, K.A. Martin, and H.H. Suarez. 1995. Recurrent excitation in neocortical circuits. Science 269:981-985.

Freeman, W.J. 1975. Mass Action in the Nervous System. New York: Academic Press.

Gupta A., Y. Wang, and H. Markram. 2000. Organizing principles for a diversity of GABAergic interneurons and synapses in the neocortex. Science 287:273-278.

Häusler, S., and W. Maass. 2005. A statistical analysis of information processing properties of lamina-specific cortical microcircuit models, submitted. Available online as \#162 from http://www.igi.tugraz.at/maass/publications.html

Jäger, H. 2002. Tutorial on training recurrent neural networks, covering BPPT, RTRL, EKF and the "echo state network" approach. GMD Report 159. http://www.ais.fraunhofer.de/de/publ/2002.html

Jäger, H., and H. Haas 2004. Harnessing nonlinearity: Predicting chaotic systems and saving energy in wireless communication. Science 304:78-80.

Kaske, A., and W. Maass. 2005. A model for the interaction of oscillations and pattern generation with real-time computing in generic neural microcircuit models. Neural Networks, in press. Available online as \#156 from http://www.igi.tugraz.at/maass/ publications.html

Koch, C. 1999. Biophysics of Computation: Information Processing in Single Neurons. New York: Oxford Univ. Press.

Legenstein, R.A., and W. Maass. 2005a. Edge of chaos and prediction of computational power for neural microcircuit models. Submitted for publication. Available online as \#166 from http://www.igi.tugraz.at/maass/publications.html

Legenstein, R.A., and W. Maass. 2005b. What makes a dynamical system computationally powerful? In: New Directions in Statistical Signal Processing: From 
Systems to Brain, ed. S. Haykin, J.C. Principe, T.J. Sejnowski, and J.G. McWhirter. Cambridge MA: MIT Press, in press. Available online as \#165 from http://www.igi. tugraz.at/maass/publications.html

Legenstein, R.A., C. Näger, and W. Maass. 2005. What can a neuron learn with spiketiming-dependent plasticity? Neural Comp. 17:2337-2382. Available online as \#154 from http://www.igi.tugraz.at/maass/publications.html

Maass, W. 1996. Lower bounds for the computational power of networks of spiking neurons. Neural Comput. 8:1-40. Available online as \#75 from http://www.igi.tugraz.at/ maass/publications.html

Maass, W. 1997. Fast sigmoidal networks via spiking neurons. Neural Comput. 9:279304. Available online as \#82 from http://www.igi.tugraz.at/maass/ publications.html

Maass, W. 2000. On the computational power of winner-take-all. Neural Comput. 12:2519-2536. Available online as \#113 from http://www.igi.tugraz.at/maass/ publications.html

Maass W., P. Joshi, and E.D. Sontag, 2005. Principles of real-time computing with feedback applied to cortical microcircuit models. In: Advances in Neural Information Processing Systems, Cambridge MA: MIT Press, in press. Available online as \#164 from http://www.igi.tugraz.at/maass/publications.html

Maass, W., R. Legenstein, and N. Bertschinger. 2005. Methods for estimating the computational power and generalization capability of neural microcircuits. In: Advances in Neural Information Processing Systems, vol. 17, ed. L.K. Saul, Y. Weiss, and L. Bottou, pp. 865-872. Cambridge, MA: MIT Press. Available online as \#160 from http://www.igi.tugraz.at/maass/ publications.html

Maass, W., T. Natschläger, and H. Markram. 2002. Real-time computing without stable states: A new framework for neural computation based on perturbations. Neural Comput. 14:2531-2560. Available online as \#130 from http://www.igi.tugraz.at/ maass/publications.html

Maass, W., T. Natschläger, and, H. Markram. 2004. Computational models for generic cortical microcircuits. In: Computational Neuroscience: A Comprehensive Approach, ed. J. Feng, pp. 575-605. Boca Raton: Chapman and Hall/CRC. Available online as \#149 from http://www.igi.tugraz.at/maass/publications.html

Maass, W., and E.D. Sontag 2000. Neural systems as nonlinear filters. Neural Comput. 12:1743-1772. Available online as \#107 from http://www.igi.tugraz.at/maass/ publications.html

Marmarelis, P.Z., and V.Z. Marmarelis. 1978. Analysis of Physiological Systems: The White-Noise Approach. New York: Plenum Press.

Pouget, A., and, T.J. Sejnowski. 1997. Spatial transformation in the parietal cortex using basis functions. J. Cogn. Neurosci. 9:222-237.

Rieke, F., D. Warland, R. de Ruyter van Steveninck, and W. Bialek. 1997. Spikes: Exploring the Neural Code. Cambridge, MA: MIT Press.

Salinas, E., and T.J. Sejnowski. 2001. Gain modulation in the central nervous system: Where behavior, neurophysiology, and computation meet. Neuroscientist 7:430-440.

Savage, J.E. 1998. Models of Computation: Exploring the Power of Computing. Reading, MA: Addison-Wesley.

Shepherd, G.M., ed. 1990. The Synaptic Organization of the Brain. New York: Oxford Univ. Press.

Shepherd, G.M., and C. Koch. 1998. Introduction to synaptic circuits. In: The Synaptic Organization of the Brain, $4^{\text {th }}$ ed., ed. G.M. Shepherd, pp. 1-36. New York: Oxford Univ. Press. 
Silberberg, G., A. Gupta, and H. Markram. 2002. Stereotypy in neocortical microcircuits. TINS 25:227-230.

Sima, J., and P. Orponen. 2003. General-purpose computation with neural networks: A survey of complexity theoretic results. Neural Comput. 15:2727-2778.

Swindale, N.V., D. Shoham, A. Grinvald, T. Bonhoeffer, and M. Hubener. 2000. Visual cortex maps are optimized for uniform coverage. Nat. Neurosci. 3:822-826.

Thomson, A.M., and O.T. Morris. 2002. Selectivity in the inter-laminar connections made by neocortical neurons. J. Neurocytol. 31:239-246.

Tsodyks, M. 2002. Neural circuits: Models of emergent functions. In: International Encyclopedia of the Social and Behavioral Sciences, Elsevier Science, http://www. iesbs.com 